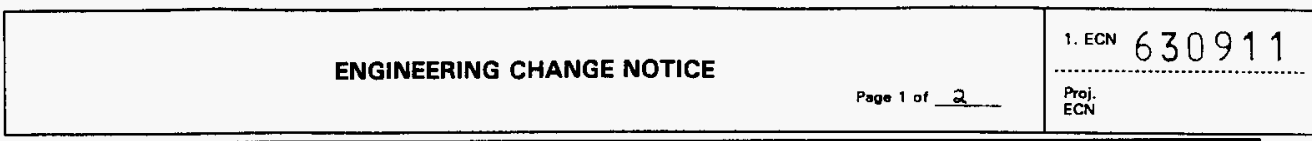

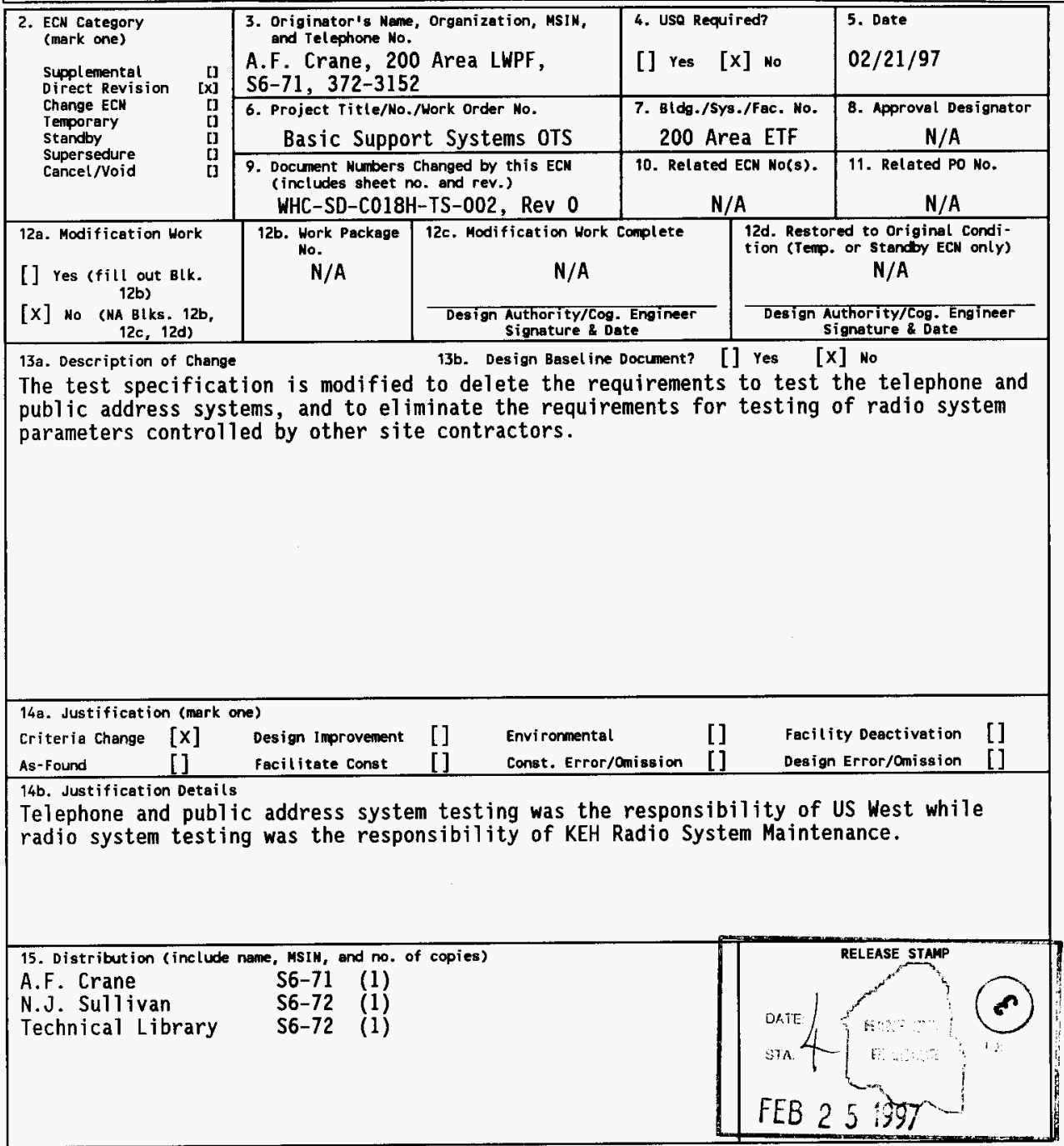

A-7900-013-2 (05/96) GEF095 


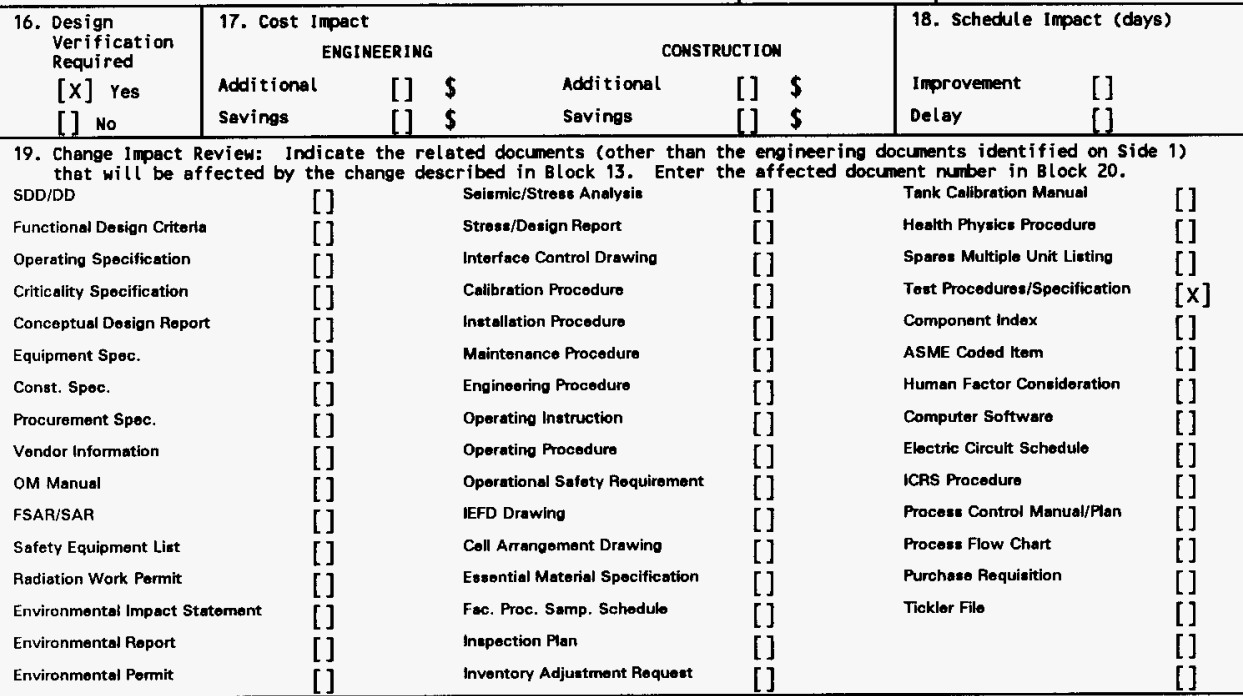

20. Other Affected Documents: (NOTE: Documents (isted below will not be revised by this ECW.) Signatures below indicate that the signing organization has been notified of other affected docunents listed below. Document Number/Revision Document Number/Revision Document Number Revision 0SP-0TP-005

21. Approvals

Signature

Design Authority A.F. Crane at Canch

Cog. Eng.

Cog. Mgr. N.J. sullivan hf ful $O P=$

QA

Safety

Environ.

other

\begin{tabular}{|c|c|c|}
\hline \multirow[t]{2}{*}{ Date } & \multirow[t]{2}{*}{ Signature } & \multirow[t]{2}{*}{ Date } \\
\hline & & \\
\hline \multirow{8}{*}{$2-21-9 ?$} & an & \\
\hline & Safety & \\
\hline & Design & \\
\hline & Environ. & \\
\hline & Other & \\
\hline & DEPARTMEUT OF ENERGY & \\
\hline & $\begin{array}{l}\text { Signature or Control Number that } \\
\text { tracks the Approval Signature }\end{array}$ & \\
\hline & ADDITIONAL & \\
\hline & 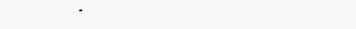 & \\
\hline & & \\
\hline
\end{tabular}




\section{Area Effluent Treatment Facility Basic Support Systems Operational Test Specification}

A. F. Crane

Rust Federal Services of Hanford Inc., Richland, WA 99352

U.S. Department of Energy Contract DE-AC06-96RL13200

EDT/ECN: 630911

Org Code: 32200

UC: 510

B\&R Code: EW3130020

Charge Code: A2093

Total Pages: $3631 \mathrm{cms} 2 / 25 / 47$

Key Words: 200 Area ETF, Basic Support Systems, Test Specification, Test Requirements

Abstract: This document identifies the test requirements for the 200 Area Effluent Treatment Facility Basic Support Systems

TRADEMARK DISCLAIMER. Reference herein to any specific commercial product, process, or service by trade name, trademark, manufacturer, or otherwise, does not necessarily constitute or imply its endorsement, recommendation, or favoring by the United States Government or any agency thereof or its contractors or subcontractors.

Printed in the United States of America. To obtain copies of this document, contact: Document Control Services, P.O. Box 950, Mailstop H6-08, Richland WA 99352, Phone (509) 372-2420;

Fax (509) 376-4989.
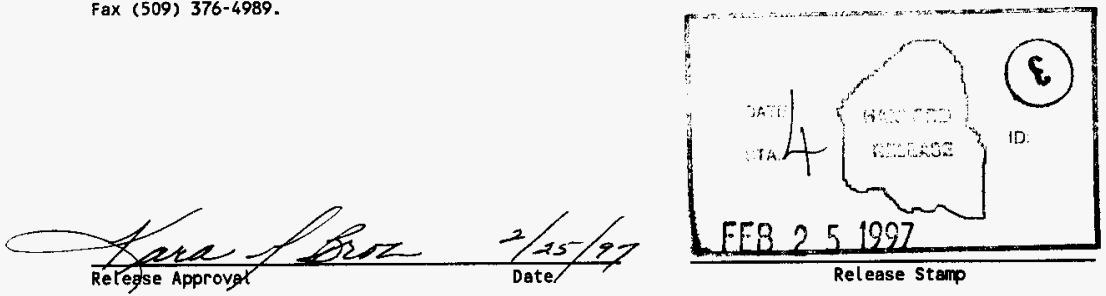
RECORD OF REVISION

(1) Docunent Number

HNE

WHE-SD-C018H-TS-

002

Page 1

(2) Title

200 Area Effluent Treatment Facility Basic Support Systems Operational Test

Specification

CHANGE CONTROL RECORD

\begin{tabular}{|c|c|c|c|}
\hline \multirow[b]{2}{*}{$\begin{array}{l}\text { Revision } \\
\text { ( }\end{array}$} & \multirow{2}{*}{$\begin{array}{l}\text { (4) Description of Chenge - Replace, Add, and Delete Pages } \\
\text { E D T }\end{array}$} & \multicolumn{2}{|c|}{ Authorized for Relesse } \\
\hline & & (5) Cog. Engr. & (6) Cog. Mgr. Date \\
\hline $1 \mathrm{RS}$ & $\begin{array}{l}\text { The test specification is modified to } \\
\text { delete the requirements to test the } \\
\text { telephone and public address systems and to } \\
\text { eliminate the requirements for testing of } \\
\text { the radio system parameters controlled by } \\
\text { other site contractors. (ECN- }-3 \text { s }_{1} \text { ) }\end{array}$ & $\underset{2.21 / 97}{102}$ & reffued $>_{2 / 21 / 97}$ \\
\hline & & & \\
\hline & & & \\
\hline & & & \\
\hline & & & \\
\hline & & & \\
\hline & & & \\
\hline & & & \\
\hline & & & \\
\hline & & & \\
\hline & & & \\
\hline & & & \\
\hline & & & \\
\hline & & & \\
\hline & & & \\
\hline & & & \\
\hline & & & \\
\hline & & & \\
\hline & & & \\
\hline & & & \\
\hline & & & \\
\hline & & & \\
\hline & & & \\
\hline & & & \\
\hline & & & \\
\hline & & & \\
\hline & & & \\
\hline & & & \\
\hline & & & \\
\hline & & & \\
\hline
\end{tabular}


HNF-SD-C018H-TS-002, Rev 1

\section{AREA EFFLUENT TREATMENT FACILITY BASIC SUPPORT SYSTEMS OPERATIONAL TEST SPECIFICATION}

\subsection{PURPOSE}

This document shall identify the test requirements for the 200 Area Effluent Treatment Facility (200 Area ETF) Basic Support Systems operational testing activities. As specified in the Design Construction Specification (DCS), $V$ C018HC1-001, contractor performed acceptance testing of the facility wili perform testing to a level consistent with actual "operational testing" as defined by WHC-CM-6.1, EP-4.2. As a result, the required level of detai1 for WHC performed operational testing is lessened. Specifically, the purpose of operational testing will be to perform any additional testing deemed important in fully defining operational characteristics of the systems. If all test requirements 1 isted in this document have been satisfied prior to facility turnover, no Operational Test Procedure will be required. Test Requirements may be satisfied by contractor performed testing or justified as not requiring testing by the cognizant engineer.

This test specification identifies the operational testing which demonstrates functional, operational and design requirements of the Basic Support Systems have been met. The Basic Support Systems include:

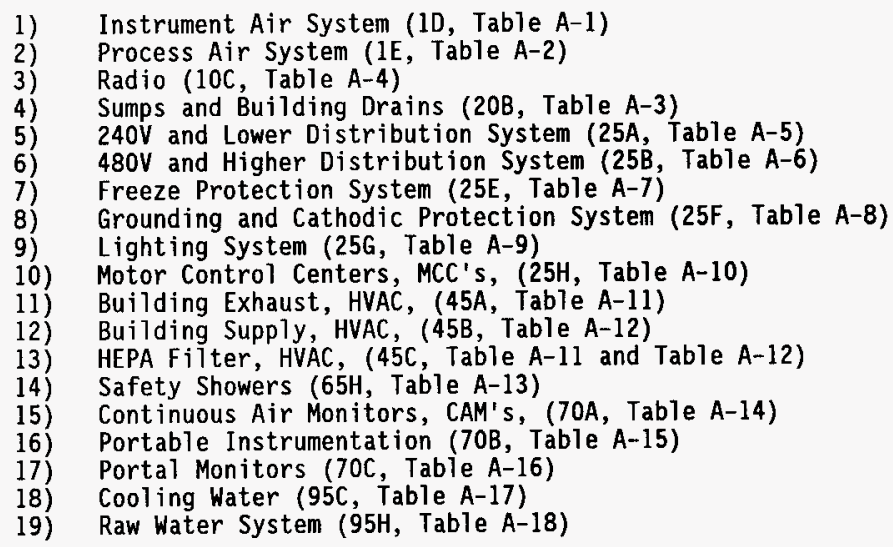

Testing of the telephone and public address systems performed by other site contractors is not included within this document. 
HNF-SD-C018H-TS-002, Rev 1

\subsection{INITIAL FACILITY CONDITIONS}

Testing will be conducted as the individual subsystems become operational to demonstrate the operability of the Basic Support Systems. The following list contains the initial conditions which must be met to perform testing of the Basic Support Systems:

1) Instrument Air System (10)

- MCS testing completed to the extent required to allow performance of this system test.

- Electrical system is in service to the extent required to allow performance of this system test.

2) Process Air System (1E)

- MCS testing completed to the extent required to allow performance of this system test.

- Electrical system is in service to the extent required to allow performance of this system test.

3) Radio (10C)

Electrical system is in service to the extent required to allow performance of this system test.

4) Sumps and Building Drains (20B)

- MCS testing completed to the extent required to allow performance of this system test.

- Electrical system is in service to the extent required to allow performance of this system test.

5) 240V and Lower Distribution System (25A)

- MCS testing completed to the extent required to allow performance of this system test.

- Electrical system is in service to the extent required to allow performance of this system test.

6) $480 \mathrm{~V}$ and Higher Distribution System (25B)

MCS testing completed to the extent required to allow performance of this system test.

- Electrical system is in service to the extent required to allow performance of this system test.

7) Freeze Protection System (25E)

- Electrical system is in service to the extent required to allow performance of this system test.

8) Grounding and Cathodic Protection System (25F)

- Electrical system is in service to the extent required to allow performance of this system test.

9) Lighting System (25G)

Electrical system is in service to the extent required to allow performance of this system test.

10) Motor Control Centers, MCC's, (25H)

- MCS testing completed to the extent required to allow performance of this system test.

- Electrical system is in service to the extent required to allow performance of this system test. 


$$
\text { HNF-SD-C018H-TS-002, Rev } 1
$$

11) Building Exhaust, HVAC, (45A)

- MCS testing completed to the extent required to allow performance of this system test.

- Electrical system is in service to the extent required to allow performance of this system test.

12) Building Supply, HVAC, (45B)

- MCS testing completed to the extent required to allow performance of this system test.

- Electrical system is in service to the extent required to allow performance of this system test.

13) HEPA Filter, HVAC, (45C) Instrument Air System is in service.

- HVAC Systems completed to the extent to allow performance of this test.

14) Safety Showers (65H)

- Sanitary water available to the safety showers.

15) Continuous Air Monitors, CAM's, (70A)

- Electrical system is in service to the extent required to allow performance of this system test.

16) Portable Instrumentation (70B)

$$
\text { - Not applicable. }
$$

17) Portal Monitors (70C)

- Electrical system is in service to the extent required to allow performance of this system test.

18) Cooling Water (95C)

- MCS testing completed to the extent required to allow performance of this system test.

- Electrical system is in service to the extent required to allow performance of this system test.

19) Raw Water System (95H)

- Instrument Air System is in service.

- Raw Water supply available. 
HNF-SD-C018H-TS-002, Rev 1

\subsection{TEST REQUIREMENTS}

The technical requirements for operational testing of the Effluent Treatment Facility Basic Support Systems are defined by the test requirements presented in Appendix A. Appendix A will contain a table for each system in the module. The tables will contain the test requirements and acceptance criteria for each requirement. Space will be provided to record the document(s) used to satisfy the acceptance criteria.

These test requirements demonstrate the following:

Basic Support Systems and associated support equipment operate as designed.

As applicable, the control systems operate and status the Basic Support Systems.

Testing will utilize Operational Test Procedures to demonstrate operability of the ETF Basic Support Systems.

\subsection{Applicable Documents}

V-C018HC1-001, Design Construction Specification Project C-018H 242A

Evaporator/PUREX Plant Process Condensate Treatment Facility forms a part of the Basis of Design to the extent specified in the applicable sections of this document. In the event of conflict between documents referenced herein and the requirements of this specification, the requirements of this specification shall take precedence.

\subsection{ACCEPTANCE CRITERIA}

The reference(s) which document the completion of each acceptance criterium is provided in the Completed By column of Appendix A. If no reference document is provided, demonstration of the test requirement in an OTP may be warranted. Upon completion of the operability test for this module, the requirement/acceptance criteria will be verified by the LEF Process Engineering system cognizant engineer. The cognizant engineer will document his verification by initialing and dating the spaces provided in the Verification column of Appendix $A$. The verified appendices will be included as part of the Operability Test Report.

The LEF cognizant engineer may close out a requirement based on witnessing actions to meet acceptance criteria during the performance of an approved Adtechs test procedure. The LEF cognizant engineer may also sign a requirement if technical justification can be provided without actually performing the test. The technical justification, where required, shall be included in the Operability Test Report. 
HNF-SD-C018H-TS-002, Rev 1

4.1 Data Required:

As a minimum, those parameters called out in the acceptance criteria section of Appendix $A$ will be recorded to evaluate whether system(s) performance meets the acceptance criteria. 


\begin{tabular}{|c|c|c|c|c|}
\hline \multicolumn{5}{|c|}{ Appendix A, Table A-1, Instrument Air System (ID) } \\
\hline$\#$ & Test Requirement & Acceptance Criteria & $\begin{array}{l}\text { Completion Reqd } \\
\text { By (Document) }\end{array}$ & $\begin{array}{l}\text { Verification } \\
\text { Initials/Date } \\
\end{array}$ \\
\hline 1 & $\begin{array}{l}\text { Verify proper operation of the } \\
\text { instrument air low pressure alarm. }\end{array}$ & $\begin{array}{l}\text { The low pressure alarm PAL-1D011 is } \\
\text { activated when the pressure is } 80 \pm 2 \\
\text { PSIG }\end{array}$ & $\begin{array}{l}\text { ATP } S-1231-405 \\
\text { Step } 7.58-7.64\end{array}$ & \\
\hline 2 & $\begin{array}{l}\text { Verify Instrument Air System can be } \\
\text { operated in the Automatic and Manual } \\
\text { modes as selected from the MCS. }\end{array}$ & $\begin{array}{l}\text { The AUTO/MANUAL position of HS-1004l } \\
\text { shall be initiated at MCS. }\end{array}$ & $\begin{array}{l}\text { CAI0 S-1231-318 } \\
\text { Sep } 6.24 \& 6.25 \\
\text { S-1231-405 } \\
\text { Sect. } 7.0 \\
\end{array}$ & \\
\hline 3 & $\begin{array}{l}\text { Verify operating logics of the } \\
\text { instrument air dryer. }\end{array}$ & $\begin{array}{l}\text { The dryer unit alternates in service } \\
\text { tower at intervals as set per dryer } \\
\text { timer. }\end{array}$ & $\begin{array}{l}\text { ATP S-1231-405 } \\
\text { Step } 7.6-7.25\end{array}$ & \\
\hline 4 & $\begin{array}{l}\text { Verify that the following solenoid } \\
\text { drain valves function properly per } \\
\text { the set cycle of } 45 \text { minutes close and } \\
5 \text { seconds open. (See attached list of } \\
\text { solenoid drain valves) }\end{array}$ & $\begin{array}{l}\text { Verify each valve in attached list } \\
\text { operates as per the given logic. }\end{array}$ & $\begin{array}{l}\text { ATP } S-1231-405 \\
\text { Step } 7.39-7.50\end{array}$ & \\
\hline 5 & $\begin{array}{l}\text { Verify proper operation of system } \\
\text { regulating valves. }\end{array}$ & $\begin{array}{l}\text { The PCV-1D031 has been set at } 90 \pm 2 \\
\text { PSIG and maintain header pressure } \\
\text { under load conditions. }\end{array}$ & $\begin{array}{c}\text { ATP S-1231-405 } \\
\text { Step } 7.52- \\
7.57 \\
\end{array}$ & \\
\hline 6 & $\begin{array}{l}\text { Verify setpoint of system relief } \\
\text { valves. }\end{array}$ & $\begin{array}{l}\text { PSV-1D030 has been tested and set at } \\
175 \pm 2 \text { PSIG. Certification or bench } \\
\text { test data is available. }\end{array}$ & & \\
\hline 7 & $\begin{array}{l}\text { Verify drying capability of the } \\
\text { instrument air dryers. }\end{array}$ & $\begin{array}{l}\text { The instrument air dryer system } \\
\text { provides dry air with dew point } \\
\leq-40^{\circ} \mathrm{F} \text { at } \geq 50 \% \text { of full load flow. }\end{array}$ & $\begin{array}{l}\text { S-1231-405 } \\
\text { STEP } 7.31-7.38\end{array}$ & \\
\hline 8 & $\begin{array}{l}\text { Verify proper system operation at } \\
\text { loaded conditions. }\end{array}$ & $\begin{array}{l}\text { System provides dry air at a pressure } \\
\text { of }>90 \text { PSIG when the plant is at } \\
\text { ful } 1 \text { operating conditions. }\end{array}$ & & \\
\hline
\end{tabular}


HNF-SD-C018H-TS-002, REV . I

\begin{tabular}{|c|c|c|c|c|}
\hline \multicolumn{5}{|c|}{ Appendix A, Table A-2, Service Air System (IE) } \\
\hline$\#$ & Test Requirement & Acceptance Criteria & $\begin{array}{l}\text { Completion Reqd } \\
\text { By (Document) }\end{array}$ & $\begin{array}{l}\text { Verification } \\
\text { Initials/Date } \\
\end{array}$ \\
\hline 1 & $\begin{array}{l}\text { Verify the operation of the system } \\
\text { low pressure alarm. }\end{array}$ & $\begin{array}{l}\text { The low pressure al arm PAL-1B011 is } \\
\text { activated when the pressure is } 80 \pm 2 \\
\text { PSIG }\end{array}$ & $\begin{array}{l}\text { S-1231-404 } \\
\text { STEP } 7.2 .88\end{array}$ & \\
\hline 2 & $\begin{array}{l}\text { Verify Service Air System can be } \\
\text { operated in the Automatic and Manual } \\
\text { modes as selected from the MCS. }\end{array}$ & $\begin{array}{l}\text { The AUTO/MANUAL position of HS-1B030 } \\
\text { shall be initiated at MCS. }\end{array}$ & $\begin{array}{l}\text { S-1231-404 } \\
\text { SECT. } 7.3 \& 7.4\end{array}$ & \\
\hline 3 & $\begin{array}{l}\text { Verify that the following solenoid } \\
\text { drain valves function properly per } \\
\text { the set cycle of } 45 \text { minutes close and } \\
5 \text { seconds open. }\end{array}$ & $\begin{array}{l}\text { Verify each valve operates as per the } \\
\text { given logic. }\end{array}$ & $\begin{array}{l}\text { S-1231-404 } \\
\text { STEPS } 7.2 .68- \\
7.2 .80\end{array}$ & \\
\hline 4 & $\begin{array}{l}\text { Verify proper operation of system } \\
\text { pressure regulating valves. }\end{array}$ & $\begin{array}{l}\text { The PCV }-1 B 037 \text { and PCV }-1 B 038 \text { have been } \\
\text { set at } 90 \pm 2 \text { PSIG and maintain } \\
\text { header pressure under load } \\
\text { conditions. }\end{array}$ & $\begin{array}{l}\text { S-1231-404 } \\
\text { STEPS } 7.2 .50- \\
7.2 .54\end{array}$ & \\
\hline 5 & $\begin{array}{l}\text { Verify proper operation of the } \\
\text { service air system. }\end{array}$ & $\begin{array}{l}\text { The system provides service air at a } \\
\text { pressure of }>90 \text { PSIG when the plant } \\
\text { is at full operating conditions. } \\
\end{array}$ & & \\
\hline 6 & $\begin{array}{l}\text { Verify proper loading of service air } \\
\text { compressor. }\end{array}$ & $\begin{array}{l}\text { The compressor loads at } 110 \pm 5 \text { PSIG } \\
\text { and unloads at } 120 \pm 5 \text { PSIG. }\end{array}$ & $\begin{array}{c}\text { S-1231-404 } \\
\text { STEPS } 7.2 .44 \& \\
7.2 .53 \\
\end{array}$ & \\
\hline 7 & $\begin{array}{l}\text { Verify that no unusual noise or } \\
\text { vibration of the compressor during } \\
\text { startup, operation or shutdown. }\end{array}$ & $\begin{array}{l}\text { Vibration data taken and compressor } \\
\text { observed during system operation. }\end{array}$ & $\begin{array}{c}\text { S-1231-404 } \\
\text { STEPS } 7.2 .3 \& \\
7.2 .32 \\
\end{array}$ & \\
\hline
\end{tabular}


HNF-SD-C018H-TS-002, REV. 1

\begin{tabular}{|c|c|c|c|c|}
\hline \multicolumn{5}{|c|}{ Appendix A, Table A-3, Radio System (10C) } \\
\hline$\#$ & Test Requirement & Acceptance Criteria & $\begin{array}{l}\text { Completion Reqd } \\
\text { By (Document) }\end{array}$ & $\begin{array}{l}\text { Verification } \\
\text { Initials/Date } \\
\end{array}$ \\
\hline 1 & $\begin{array}{l}\text { Range and coverage of transmitter is } \\
\text { sufficient to provide coverage at } \\
\text { SALDS, all TEDF equipment locations, } \\
\text { LERF, and within the facility. All } \\
\text { areas of non-coverage must be deemed } \\
\text { acceptable to facility management. }\end{array}$ & $\begin{array}{l}\text { Establish radio communications in all } \\
\text { equipment/process rooms within ETF, } \\
\text { as well as outside process areas and } \\
\text { SALDS location. }\end{array}$ & & \\
\hline 2 & $\begin{array}{l}\text { Verify that radios can be operated in } \\
\text { the plant without interfering with } \\
\text { equipment/system controls. }\end{array}$ & $\begin{array}{l}\text { Determine that base transmitter or } \\
\text { portable units do not cause } \\
\text { detrimental interference with plant } \\
\text { control systems, ancillary } \\
\text { instrumentation or with the fire } \\
\text { suppression system (radio link to } \\
\text { Hanford Fire Department). }\end{array}$ & & \\
\hline
\end{tabular}


HNF-SD-C018H-TS-002, REV . 1

\begin{tabular}{|c|c|c|c|c|}
\hline \multicolumn{5}{|c|}{ Appendix A, Table A-4, Sumps and Building Drains System (20B) } \\
\hline$\#$ & Test Requirement & Acceptance Criteria & $\begin{array}{c}\text { Completion Reqd } \\
\text { By (Document) }\end{array}$ & $\begin{array}{l}\text { Verification } \\
\text { Initials/Date }\end{array}$ \\
\hline 1 & $\begin{array}{l}\text { Alarms and switches associated with } \\
\text { Sump System } 20 \mathrm{~B} \text { actuate at the given } \\
\text { setpoints. }\end{array}$ & $\begin{array}{l}\text { Setpoints as listed in } 5-1223-003 \\
\text { Rev. 2, Set-Value Selection Basis. }\end{array}$ & $S-1231-100$ & \\
\hline 2 & $\begin{array}{l}\text { Verify pump motors and pumps operate } \\
\text { without excessive vibration, noise or } \\
\text { overheating. }\end{array}$ & $\begin{array}{l}\text { Monitor and record data for vibration } \\
\text { and bearing temperatures for } \\
\text { evaluation of pump and motor } \\
\text { operation. }\end{array}$ & $\begin{array}{c}\text { S-1231-306 S- } \\
1231-410 \text { STEPS } \\
7.2 .16-7.3 .16 \\
\text { S-1231-411 } \\
7.2 .16-7.3 .16\end{array}$ & \\
\hline 3 & $\begin{array}{l}\text { Verify sump pumps } 20 \mathrm{~B}-\mathrm{P}-1 \mathrm{~A} / \mathrm{B} \text { operate } \\
\text { with normal motor current. }\end{array}$ & $\begin{array}{l}\text { Sump Pumps (20B-P-1A/B) motor current } \\
\text { does not exceed } 10.2 \text { amps as } \\
\text { indicated on II-20B032 and II-20B034 } \\
\text { respectively. }\end{array}$ & $\begin{array}{l}\text { S-1231-306 S- } \\
1231-410 \text { STEP } \\
7.2 .107 .3 .10\end{array}$ & \\
\hline 4 & $\begin{array}{l}\text { Verify sump pumps } 20 B-P-2 A / B \text { operate } \\
\text { with normal motor current. }\end{array}$ & $\begin{array}{l}\text { Sump Pumps (20B-P-2A/B) motor current } \\
\text { does not exceed } 12.5 \text { amps as } \\
\text { indicated on II-20B034 and II-20B036 } \\
\text { respectively }\end{array}$ & $\begin{array}{l}\text { S-1231-306 S- } \\
1231-411 \text { STEP } \\
7.2 .107 .3 .10\end{array}$ & \\
\hline 5 & $\begin{array}{l}\text { Verify proper seal water flow } \\
\text { established for Sump Pump 20B-P-1A/B. }\end{array}$ & $\begin{array}{l}\text { Flow indicator } 60 \mathrm{H}-129 \text { Sump Pump 20B- } \\
\text { P-1A/B Seal Water Flow calibrated for } \\
\geq 4 \mathrm{gpm}\end{array}$ & $\begin{array}{l}\text { S-1231-410 STEP } \\
7.2 .6,7.3 .6\end{array}$ & \\
\hline 6 & $\begin{array}{l}\text { Verify proper seal water flow } \\
\text { established for Sump Pump 20B-P-2A/B. }\end{array}$ & $\begin{array}{l}\text { Flow indicator } 60 \mathrm{H}-136 \text { Sump Pump 20B- } \\
\text { P-2A/B Seal Water Flow calibrated for } \\
2-4 \text { gpm }\end{array}$ & $\begin{array}{l}S-1231-411 \text { STEP } \\
7.2 .6,7.3 .6\end{array}$ & \\
\hline 7 & Verify MCS indication from AI-20B021. & $\begin{array}{l}\text { Sump Tank } 20 \mathrm{~B}-\mathrm{TK}-1 \text { Conductivity meter } \\
\mathrm{AI}-20 \mathrm{~B} 021 \text { reads between } 0-1500 \mathrm{E}-6 \\
\mathrm{~S} / \mathrm{Cm} \text { at the MCS }\end{array}$ & $\begin{array}{c}\text { S-1231-410 STEP } \\
7.7 .14\end{array}$ & \\
\hline 8 & Verify MCS indication from AI-20B024. & $\begin{array}{l}\text { Sump Tank 20B-TK-2 Conductivity meter } \\
\text { AI-20B024 reads between } 0-1500 E-6 \\
\text { S/cm at the MCS }\end{array}$ & $\begin{array}{c}\mathrm{S}-1231-411 \text { STEP } \\
7.6 .14\end{array}$ & \\
\hline
\end{tabular}


HNF-SD-C018H-TS-002, REV. 1

\begin{tabular}{|c|c|c|c|c|}
\hline$\#$ & Test Requirement & Acceptance Criteria & $\begin{array}{l}\text { Completion Reqd } \\
\text { By (Document) }\end{array}$ & $\begin{array}{l}\text { Verification } \\
\text { Initials/Date }\end{array}$ \\
\hline 9 & $\begin{array}{l}\text { Verify proper operation of the Sump } \\
\text { Pumps in the Automatic mode. }\end{array}$ & $\begin{array}{l}\text { When in the AUTO mode the Sump Pumps } \\
\text { and controlling software operate as } \\
\text { defined by the latest revision of } \\
\text { logic diagram } \mathrm{H}-288986\end{array}$ & $\begin{array}{c}\text { S- } 1231-410 \text { SECT } \\
7.7 \\
\text { S-1231-411 SECT } \\
7.6, S-1231-100 \\
\end{array}$ & \\
\hline 10 & $\begin{array}{l}\text { Verify proper operation of the Sump } \\
\text { Pumps in the Manual mode. }\end{array}$ & $\begin{array}{l}\text { When in the MANUAL mode the Sump } \\
\text { Pumps and controlling software } \\
\text { operate as defined by the latest } \\
\text { revision of logic diagram } \mathrm{H}-288986\end{array}$ & $\begin{array}{c}\text { S-1231-410 \& } \\
411 \text { SECT. } 7.2 \\
7.3,7.4 \\
\text { S- } 1231-100\end{array}$ & \\
\hline 11 & $\begin{array}{l}\text { Verify drainage pumps } 20 \mathrm{~B}-\mathrm{P}-3 / 4 / 5 / 6 \\
\text { operate without any unusual noise or } \\
\text { vibration. }\end{array}$ & $\begin{array}{l}\text { Observe pumps during operation for } \\
\text { usual noise or indications of unusual } \\
\text { vibration. }\end{array}$ & & \\
\hline 12 & $\begin{array}{l}\text { Verify operation of pumps } 20 \mathrm{~B}-\mathrm{P}- \\
3 / 4 / 5 / 6 \text { using local controls. }\end{array}$ & $\begin{array}{l}\text { Drainage pumps } 20 \mathrm{~B}-\mathrm{P}-3 / 4 / 5 / 6 \text { start } \\
\text { when actuated by their respective } \\
\text { handswitches } \mathrm{HS}-20 \mathrm{~B} 050 \text {, } \mathrm{HS}-20 \mathrm{BO} 051 \text {, } \\
\mathrm{HS}-20 \mathrm{~B} 017 \text { and } \mathrm{HS}-20 \mathrm{~B} 018\end{array}$ & $\begin{array}{l}\text { S-1231-412 } \\
\text { STEPS 7.2.5, } \\
7.3 .5,7.4 .5 \\
7.5 .5\end{array}$ & \\
\hline 13 & $\begin{array}{l}\text { Verify operation of control circuitry } \\
\text { for pumps } 20 \mathrm{~B}-\mathrm{P}-1 \mathrm{~A} / \mathrm{B} \text { and } 20 \mathrm{~B}-\mathrm{P}-2 \mathrm{~A} / \mathrm{B} \text {. }\end{array}$ & $\begin{array}{l}\text { Control loop } 208001 \text { and } 20 \mathrm{~B} 002 \\
\text { actuate the correct pump control } \\
\text { response }\end{array}$ & $\begin{array}{l}\text { S-1231-410 \& } \\
411 \text { SECTION } 7.7 \\
\text { S-1231-100 }\end{array}$ & \\
\hline
\end{tabular}


HNF-SD-C018H-TS-002, REV. 1

\begin{tabular}{|c|l|l|l|l|}
\hline \multicolumn{3}{|c|}{ Appendix A, Table A-5, 240V and Lower Distribution System (25A) } \\
\hline$\#$ & Test Requirement & Acceptance Criteria & $\begin{array}{c}\text { Completion Reqd } \\
\text { By (Document) }\end{array}$ & $\begin{array}{c}\text { Verification } \\
\text { Initials/Date }\end{array}$ \\
\hline 1 & Note 1 & & \\
\hline
\end{tabular}

Note 1: All testing of the electrical systems has been performed during construction testing. No further testing is required. 
HNF-SD-C018H-TS-002, REV. 1

\begin{tabular}{|c|l|l|l|l|}
\hline \multicolumn{3}{|c|}{ Appendix A, Table A-6, 480V and Higher Distribution System (25B) } \\
\hline$\#$ & Test Requirement & Acceptance Criteria & $\begin{array}{c}\text { Completion Reqd } \\
\text { By (Document) }\end{array}$ & $\begin{array}{c}\text { Verification } \\
\text { Initials/Date }\end{array}$ \\
\hline 1 & Note 1 & & \\
\hline
\end{tabular}

Note 1: All testing of the electrical systems has been performed during construction testing. No further testing is required. 
HNF-SD-C018H-TS-002, REV. 1

\begin{tabular}{|c|c|c|c|c|}
\hline \multicolumn{5}{|c|}{ Appendix A, Table A-7, Freeze Protection System (25E) } \\
\hline$\#$ & Test Requirement & Acceptance Criteria & $\begin{array}{l}\text { Completion Reqd } \\
\text { By (Document) }\end{array}$ & $\begin{array}{l}\text { Verification } \\
\text { Initials/Date }\end{array}$ \\
\hline 1 & $\begin{array}{l}\text { Heat Tracing is installed properly } \\
\text { with insulation and cables (and } \\
\text { associated equipment) having no } \\
\text { physical damage. }\end{array}$ & $\begin{array}{l}\text { Megger test with resistance not less } \\
\text { than } 10 \mathrm{M} \Omega \text {. }\end{array}$ & $\begin{array}{l}\text { S-1231-001 } \\
\text { Sect } 7.0\end{array}$ & \\
\hline 2 & $\begin{array}{l}\text { Verify proper calibration of heat } \\
\text { trace channels. }\end{array}$ & $\begin{array}{l}\text { All channels are calibrated; for both } \\
\text { the standard mode and the narrow } \\
\text { range mode per instructions in } \\
\text { Chromalox user's manual 0037-75087, p } \\
24-26 \text {. }\end{array}$ & $\begin{array}{l}\text { S-1231-001 } \\
\text { Sect } 7.0\end{array}$ & \\
\hline 3 & $\begin{array}{l}\text { Verify Heat Tracing maintains systems } \\
\text { at the correct temperatures. }\end{array}$ & $\begin{array}{l}\text { Temperature set points are correct } \\
\text { according to pipe contents: eg. } 70^{\circ} \mathrm{F} \\
\text { for } \mathrm{NaOH}, 40^{\circ} \mathrm{F} \text { for process influent } \\
\text { and effluent, } 5^{\circ} \text { for } \mathrm{H}_{2} \mathrm{SO}_{4} \text {. } \\
\end{array}$ & $\begin{array}{l}\text { S-1231-001 } \\
\text { Sect } 7.0\end{array}$ & \\
\hline 4 & $\begin{array}{l}\text { Verify proper alarm response for each } \\
\text { heat trace circuit. }\end{array}$ & $\begin{array}{l}\text { Alarm set points for each circuit are } \\
\text { appropriate for pipe content and } \\
\text { deadband setting; the alarm set } \\
\text { points are not within the range of } \\
\text { the deadband. Refer to submittals } \\
\# V-1381-007-301 \text { through } \# V-1381-007- \\
314 \text {. }\end{array}$ & $\begin{array}{r}\text { S-1231-338 } \\
\text { Sect } 6.0\end{array}$ & \\
\hline 5 & $\begin{array}{l}\text { Verify output update rate for each } \\
\text { heat trace circuit. }\end{array}$ & $\begin{array}{l}\text { Output update rate is set at least } 30 \\
\text { seconds. }\end{array}$ & $\begin{array}{l}\text { S-1231-001 } \\
\text { Sect } 7.0\end{array}$ & \\
\hline 6 & $\begin{array}{l}\text { Verify that circuits are labeled } \\
\text { correctly. }\end{array}$ & $\begin{array}{l}\text { Each circuit label corresponds to the } \\
\text { pipe }(s) \text { that they protect. }\end{array}$ & $\begin{array}{r}S-1231-001 \\
\text { Sect } 7.0 \\
\end{array}$ & \\
\hline
\end{tabular}


HNF-SD-C018H-TS-002, REV. 1

\begin{tabular}{|c|c|c|c|c|}
\hline \multicolumn{5}{|c|}{ Appendix A, Table A-8, Grounding and Cathodic Protection System (25F) } \\
\hline$\#$ & Test Requirement & Acceptance Criteria & $\begin{array}{c}\text { Completion Reqd } \\
\text { By (Document) }\end{array}$ & $\begin{array}{l}\text { Verification } \\
\text { Initials/Date }\end{array}$ \\
\hline 1 & Note 1 & & & \\
\hline
\end{tabular}

Note 1: All testing of the electrical systems has been performed during construction testing. No further testing is required. 
HNF-SD-C018H-TS-002, REV. 1

\begin{tabular}{|c|c|c|c|c|}
\hline \multicolumn{5}{|c|}{ Appendix A, Table A-9, Lighting System (25G) } \\
\hline$\#$ & Test Requirement & Acceptance Criteria & $\begin{array}{l}\text { Completion Reqd } \\
\text { By (Document) }\end{array}$ & $\begin{array}{l}\text { Verification } \\
\text { Initials/Date }\end{array}$ \\
\hline 1 & $\begin{array}{l}\text { Verify proper operation of emergency } \\
\text { lights in response to a loss of } \\
\text { power. }\end{array}$ & \multicolumn{3}{|c|}{$\begin{array}{l}\text { See specification WHC-SD-C018H-TS-008, } 200 \text { Area Effluent Treatment } \\
\text { Facility Loss of Plant Electrical Operational Test Specification. }\end{array}$} \\
\hline
\end{tabular}


HNF-SD-C018H-TS-002, REV. 1

\begin{tabular}{|c|l|l|l|l|}
\hline \multicolumn{3}{|c|}{ Appendix A, Table A-10, Motor Control Centers (25H) } \\
\hline$\#$ & Test Requirement & Acceptance Criteria & $\begin{array}{c}\text { Completion Reqd } \\
\text { By (Document) }\end{array}$ & $\begin{array}{c}\text { Verification } \\
\text { Initials/Date }\end{array}$ \\
\hline 1 & Note 1 & & \\
\hline
\end{tabular}

Note 1: All testing of the electrical systems has been performed during construction testing. No further testing is required. 
HNF-SD-C018H-TS-002, REV. 1

\begin{tabular}{|c|c|c|c|c|}
\hline \multicolumn{5}{|c|}{ Appendix A, Table A-11, RCA HVAC System $(45 A, B, C)$} \\
\hline$\#$ & Test Requirement & Acceptance Criteria & $\begin{array}{l}\text { Completion Reqd } \\
\text { By (Document) }\end{array}$ & $\begin{array}{l}\text { Verification } \\
\text { Initials/Date }\end{array}$ \\
\hline 1 & $\begin{array}{l}\text { Verify proper operation of RCA HVAC } \\
\text { MCS indication. }\end{array}$ & $\begin{array}{l}\text { RCA HVAC instrumentation which } \\
\text { indicate a value on the OCS per P\&ID } \\
\text { H-2-89021 Sh. } 1 \text { of } 2 \text { has been } \\
\text { verified to indicate properly. }\end{array}$ & $S-1231-100$ & \\
\hline 2 & $\begin{array}{l}\text { All switches and alarms associated } \\
\text { with RCA HVAC instrumentation have } \\
\text { been verified to actuate at design set } \\
\text { values/alarm points. }\end{array}$ & $\begin{array}{l}\text { Setpoints verified as } 1 \text { isted in S- } \\
1223-003 \text {. }\end{array}$ & $S-1231-100$ & \\
\hline 3 & $\begin{array}{l}\text { Verify proper operation of RCA HVAC } \\
\text { dampers. }\end{array}$ & $\begin{array}{l}\text { RCA HVAC Dampers are capable of } \\
\text { being stroked fully open and fully } \\
\text { closed. }\end{array}$ & S-1231-401 & \\
\hline 4 & $\begin{array}{l}\text { The RCA HVAC fans operate at listed } \\
\text { design flowrates with no evidence of } \\
\text { abnormal operating characteristics, } \\
\text { including high vibration, surge or } \\
\text { high bearing temperature: }\end{array}$ & $\begin{array}{l}45 B-F-1 A \text { (RCA Supply Fan A) } 40,000 \\
\text { CFM } \pm 10 \% \text { (RCA Supply Fan B) } 40,000 \\
45 B-F-1 B \text { (RCA S } \\
\text { CFM } \pm 10 \% \text { (Vapor Compressor Room Fan) } \\
45 B-F-5 \quad 3000 \text { CFM } \\
4000 \text { CFM } \pm 10 \% \\
45 B-F-6 \text { (Dryer Room Fan) } 30 \% \\
\pm 10 \% \\
45 A-F-1 A \text { (RCA Exhaust Fan A) } 29000 \\
\text { CFM } \pm 10 \% \\
45 A-F-1 B \text { (RCA Exhaust Fan B) } 29000 \\
\text { CFM } \pm 10 \% \\
45 A-F-1 C \text { (RCA Exhaust Fan C) } 29000 \\
\text { CFM } \pm 10 \%\end{array}$ & $\begin{array}{l}\text { S-1231-308 } \\
\text { S-1231-401, } \\
7.1 .7 .32 \\
\text { S-1231-401, } \\
7.1 .11 .32 \\
\text { S-1231-401, } \\
7.1 .18 .7 \\
\text { S-1231-401, } \\
7.1 .19 .7 \\
\text { S-1231-401, } \\
7.1 .3 .55 \\
\text { S-1231-401, } \\
7.1 .4 .50 \\
\text { S-1231-401, } \\
7.1 .5 .50\end{array}$ & \\
\hline
\end{tabular}


HNF-SD-CO18H-TS-002, REV. 1

\begin{tabular}{|c|c|c|c|c|}
\hline \multicolumn{5}{|c|}{ Appendix A, Table A-11, RCA HVAC System $(45 A, B, C)$} \\
\hline \# & Test Requirement & Acceptance Criteria & $\begin{array}{l}\text { Completion Reqd } \\
\text { By (Document) }\end{array}$ & $\begin{array}{l}\text { Verification } \\
\text { Initials/Date }\end{array}$ \\
\hline 5 & $\begin{array}{l}\text { Verify fan motor operation with normal } \\
\text { motor current. }\end{array}$ & $\begin{array}{l}\text { The following fan motors do not } \\
\text { exceed their rated full load } \\
\text { amperage rating during normal } \\
\text { operation: } \\
45 B-F-1 A \text { (RCA Supply Fan A) } \\
45 B-F-1 B \text { (RCA Supply Fan B) } \\
45 B-F-5 \text { (Vapor Compressor Room Fan) } \\
45 B-F-6 \text { (Dryer Room Fan) } \\
45 A-F-1 A \text { (RCA Exhaust Fan A) } \\
45 A-F-1 B \text { (RCA Exhaust Fan B) } \\
45 A-F-1 C \text { (RCA Exhaust Fan C) }\end{array}$ & $\begin{array}{l}S-1231-308 \text { S- } \\
1231-401 \\
7.1 \cdot 3 \cdot 20 \\
7.1 .4 .16 \\
7.1 .5 .15 \\
7.1 .7 .10 \\
7.1 .11 .9\end{array}$ & \\
\hline 6 & $\begin{array}{l}\text { Verify flows have been balanced within } \\
\text { the duct system in accordance with } \\
\text { ASHRAE Handbook and AABC. }\end{array}$ & $\begin{array}{l}\text { Acceptable quantities are } \pm 10 \% \text { of } \\
\text { those shown on Drawing } \mathrm{H}-2-89021 \mathrm{Sh} \text {. } \\
1 \text { of } 2 \text { and } \mathrm{H}-2-89325 \text {. }\end{array}$ & $\begin{array}{l}S-1231-401 \\
7.2 .2 .4 \text { (AIR } \\
\text { FLOW ONLY) }\end{array}$ & \\
\hline 7 & Verify RCA space temperature control. & $\begin{array}{l}\text { RCA space temperature is controlled } \\
\text { at setpoint } \pm 4^{\circ} \mathrm{F} \text { in the cooling } \\
\text { mode and in the heating mode. }\end{array}$ & $\begin{array}{l}5-1231-401 \\
7.14,7.13, \\
7.1 .9,7.1 .10, \\
7.1 .18 .18, \\
7.1 .19 .18\end{array}$ & \\
\hline 8 & $\begin{array}{l}\text { Verify pressure control in the } \\
\text { Dryer/Drum Handling Area (Zone II). }\end{array}$ & $\begin{array}{l}\text { Controlled at a negative pressure } \\
\text { with respect to Zone III (RCA) } \\
\text { pressure. }\end{array}$ & $\begin{array}{l}\text { Recorded in S- } \\
1231-401 \\
7.1 .23 .23\end{array}$ & \\
\hline 9 & $\begin{array}{l}\text { Verify pressure control in RCA (Zone } \\
\text { III) }\end{array}$ & $\begin{array}{l}\text { RCA pressure is a negative pressure } \\
\text { with respect to outside atomospheric } \\
\text { pressure. }\end{array}$ & $\begin{array}{l}5-1231-401 \\
7.1 .16 .19 \\
7.1 .23 .17 \\
\end{array}$ & \\
\hline 10 & $\begin{array}{l}\text { Verify negative pressure maintained in } \\
\text { lab hood ducting. }\end{array}$ & $\begin{array}{l}\text { The lab hood exhaust ductwork from } \\
\text { the three lab hood fans to the main } \\
\text { RCA exhaust duct has been } \\
\text { successfully leak tested, or no } \\
\text { leakage is observed when a } \\
\text { detectable smoke is injected into } \\
\text { the lab hoods. }\end{array}$ & & \\
\hline
\end{tabular}


HNF-SD-C018H-TS-002, REV. I

\begin{tabular}{|c|c|c|c|c|}
\hline \multicolumn{5}{|c|}{ Appendix A, Table A-11, RCA HVAC System $(45 A, B, C)$} \\
\hline$\#$ & Test Requirement & Acceptance Criteria & $\begin{array}{l}\text { Completion Reqd } \\
\text { By (Document) }\end{array}$ & $\begin{array}{l}\text { Verification } \\
\text { Initials/Date }\end{array}$ \\
\hline 11 & $\begin{array}{l}\text { Verify proper operation of the RCA } \\
\text { HVAC System controls. }\end{array}$ & $\begin{array}{l}\text { The RCA HVAC System operation is } \\
\text { controlled in accordance with Logic } \\
\text { Diagram H-2-89888. }\end{array}$ & $\begin{array}{l}S-1231-100 \\
S-1231-401 \text { SECT } \\
7.1 .15-7.1 .17 \\
7.1 .23\end{array}$ & \\
\hline 12 & Verify DOP testing of HEPA filters. & $\begin{array}{l}\text { HEPA Filter DOP testing has been } \\
\text { successfully completed. }\end{array}$ & $\begin{array}{l}S-1231-401 \\
7.2 \cdot 3 \cdot 4 \\
\end{array}$ & \\
\hline 13 & $\begin{array}{l}\text { Perform leak testing of process } \\
\text { exhaust system. }\end{array}$ & $\begin{array}{l}\text { Leak testing of process exhaust has } \\
\text { been successfully completed in } \\
\text { accordance with the requirements of } \\
\text { ASME N509-1989 and N510-1989. }\end{array}$ & $\begin{array}{l}S-1231-401 \\
7.2 .1 .5\end{array}$ & \\
\hline 14 & $\begin{array}{l}\text { Verify proper operation of the } \\
\text { building relief damper. }\end{array}$ & $\begin{array}{l}\text { The building relief damper (ECN } \\
1371 \text { ) has been verified to relieve } \\
\text { at setpoint } \pm 0.10 \text { inwg. } \\
\text { Certification of testing is } \\
\text { available. }\end{array}$ & & \\
\hline 15 & $\begin{array}{l}\text { Verify proper response of the RCA HVAC } \\
\text { System to receipt of fire protection } \\
\text { signals. }\end{array}$ & $\begin{array}{l}\text { The following fans shut down upon } \\
\text { receiving a fire signal from the } \\
\text { Fire Alarm Control Panel: } \\
\text { 45B-F-1A (RCA Supply Fan A) } \\
\text { 45B-F-1B (RCA Supply Fan B) } \\
45 B-F-5 \text { (Vapor Compressor Room Fan) } \\
\text { 45B-F-6 (Dryer Room Fan) } \\
\text { 45A-F-1A (RCA Exhaust Fan A) } \\
45 A-F-1 B \text { (RCA Exhaust Fan B) } \\
\text { 45A-F-1C (RCA Exhaust Fan C) }\end{array}$ & $\begin{array}{l}S-1231-510 \\
7.1 .1\end{array}$ & \\
\hline 16 & $\begin{array}{l}\text { Verify ability to maintain temperature } \\
\text { in Thin Film Dryer room at design or } \\
\text { below. }\end{array}$ & $\begin{array}{l}\text { With the Thin Film Dryer operating, } \\
\text { the Thin Film Dryer room temperature } \\
\text { as indicated on TIC }-45 \mathrm{A007} \text { is } \\
\text { maintained at } 95^{\circ} \mathrm{F} \pm 4^{\circ} \mathrm{F} \text {. }\end{array}$ & & \\
\hline
\end{tabular}


HNF-SD-C018H-TS-002, REV. 1

\begin{tabular}{|c|c|c|c|c|}
\hline \multicolumn{5}{|c|}{ Appendix A, Table A-11, RCA HVAC System $(45 A, B, C)$} \\
\hline \# & Test Requirement & Acceptance Criteria & $\begin{array}{l}\text { Completion Reqd } \\
\text { By (Document) }\end{array}$ & $\begin{array}{l}\text { Verification } \\
\text { Initials/Date } \\
\end{array}$ \\
\hline 17 & $\begin{array}{l}\text { Verify ability to maintain temperature } \\
\text { in Vapor Compressor room at or below } \\
\text { design. }\end{array}$ & $\begin{array}{l}\text { With the Vapor Compressor operating, } \\
\text { the Vapor Compressor room } \\
\text { temperature as indicated on } \\
\text { TIC- } 45 A 006 \text { is maintained at } 95^{\circ} \mathrm{F} \pm \\
4^{\circ} \mathrm{F} \text {. }\end{array}$ & & \\
\hline
\end{tabular}


HNF-SD-CO18H-TS-002, REV. 1

\begin{tabular}{|c|c|c|c|c|}
\hline \multicolumn{5}{|c|}{ Appendix A, Table A-12, Non-RCA HVAC System $(45 A, B, C)$} \\
\hline \# & Test Requirement & Acceptance Criteria & $\begin{array}{l}\text { Completion Reqd } \\
\text { By (Document) }\end{array}$ & $\begin{array}{l}\text { Verification } \\
\text { Initials/Date } \\
\end{array}$ \\
\hline 1 & $\begin{array}{l}\text { Verify proper operation of Non-RCA } \\
\text { HVAC MCS indication. }\end{array}$ & $\begin{array}{l}\text { Non-RCA HVAC instrumentation which } \\
\text { indicate a value on the OCS per P\&ID } \\
\text { H-2-8802 } 1 \text { Sh. } 2 \text { of } 2 \text { has been } \\
\text { verified to indicate properly. }\end{array}$ & $S-1231-100$ & \\
\hline 2 & $\begin{array}{l}\text { All switches and alarms associated } \\
\text { with Non-RCA HVAC instrumentation have } \\
\text { been verified to actuate at design set } \\
\text { values/alarm points. }\end{array}$ & $\begin{array}{l}\text { Setpoints verified as } 1 \text { isted in S- } \\
1223-003 \text {. }\end{array}$ & $S-1231-100$ & \\
\hline 3 & $\begin{array}{l}\text { Verify proper operation of NON-RCA } \\
\text { HVAC dampers. }\end{array}$ & $\begin{array}{l}\text { Non-RCA HVAC Dampers are capable of } \\
\text { being stroked fully open and fully } \\
\text { closed. }\end{array}$ & $S-1231-402$ & \\
\hline 4 & $\begin{array}{l}\text { The listed Non-RCA HVAC fans operate } \\
\text { at listed design flowrates with no } \\
\text { evidence of abnormal operating } \\
\text { characteristics, including high } \\
\text { vibration, surge or high bearing } \\
\text { temperature: }\end{array}$ & $\begin{array}{l}\text { 45B-F-2 (Operations Area AHU Supply } \\
\text { Fan) 12,000 CFM } \pm 10 \% \\
45 B-F-3 \text { (Electrical Equipment Room } \\
\text { AHU Supply Fan) 2,000 CFM } \pm 10 \% \\
45 B-F-4 \text { (Control Room AHU Supply } \\
\text { Fan) } 8000 \text { CFM } \pm 10 \%\end{array}$ & $\begin{array}{l}\text { S-1231-308 } \\
\text { SECT.6 } \\
\text { (vibration, } \\
\text { temperature, } \\
\text { noise only) }\end{array}$ & \\
\hline 5 & $\begin{array}{l}\text { Verify fan motor operation with normal } \\
\text { motor current. }\end{array}$ & $\begin{array}{l}\text { Motors for the following fans do not } \\
\text { exceed their rated full load } \\
\text { amperage rating during normal } \\
\text { operation: } \\
45 B-F-2 \text { (Operations Area AHU Supply } \\
\text { Fan) } \\
45 B-F-3 \text { (Electrical Equipment Room } \\
\text { AHU Supply Fan) } \\
45 B-F-4 \text { (Control Room AHU Supply } \\
\text { Fan) }\end{array}$ & $\begin{array}{l}\text { S-1231-308 } \\
\text { SECT. } 6\end{array}$ & \\
\hline 6 & $\begin{array}{l}\text { Flows have been balanced within the } \\
\text { duct system in accordance with ASHRAE } \\
\text { Handbook and AABC. }\end{array}$ & $\begin{array}{l}\text { Acceptable quantities are } \pm 10 \% \text { of } \\
\text { those shown on Drawing } \mathrm{H}-2-89021 \mathrm{Sh} \text {. } \\
2 \text { of } 2 \text {. }\end{array}$ & $S-1231-401 / 402$ & \\
\hline
\end{tabular}


HNF-SD-CO18H-TS-002, REV. I

\begin{tabular}{|c|c|c|c|c|}
\hline \multicolumn{5}{|c|}{ Appendix A, Table A-12, Non-RCA HVAC System $(45 A, B, C)$} \\
\hline \# & Test Requirement & Acceptance Criteria & $\begin{array}{l}\text { Completion Reqd } \\
\text { By (Document) }\end{array}$ & $\begin{array}{l}\text { Verification } \\
\text { Initials/Date }\end{array}$ \\
\hline 7 & $\begin{array}{l}\text { Verify First Floor Operations Area } \\
\text { space temperature control. }\end{array}$ & $\begin{array}{l}\text { Space temperature is controlled at } \\
\text { setpoint } \pm 4^{\circ} \mathrm{F} \text { in the cooling mode } \\
\text { and in the heating mode. }\end{array}$ & $\begin{array}{l}\text { S-1231-402 } \\
\text { SECT.7.4 }\end{array}$ & \\
\hline 8 & $\begin{array}{l}\text { Verify First Floor Electrical } \\
\text { Equipment Area space temperature } \\
\text { control. }\end{array}$ & $\begin{array}{l}\text { Space temperature is controlled at } \\
\text { setpoint } \pm 4^{\circ} \mathrm{F} \text { in the cooling mode } \\
\text { and in the heating mode. }\end{array}$ & $\begin{array}{l}\text { S-1231-402 } \\
\text { SECT.7.4 }\end{array}$ & \\
\hline 9 & $\begin{array}{l}\text { Verify Control Area space temperature } \\
\text { control. }\end{array}$ & $\begin{array}{l}\text { Space temperature is controlled at } \\
\text { setpoint } \pm 4^{\circ} \mathrm{F} \text { in the cooling mode } \\
\text { and in the heating mode. }\end{array}$ & $\begin{array}{l}\text { S-1231-402 } \\
\text { SECT.7.4 }\end{array}$ & \\
\hline 10 & $\begin{array}{l}\text { Verify Control Area space relative } \\
\text { humidity control. }\end{array}$ & $\begin{array}{l}\text { Space humidity controlled between at } \\
\text { setpoint } \pm 5 \% \text {. }\end{array}$ & $\begin{array}{l}\text { S-1231-402 } \\
\text { SECT.7.3.11 }\end{array}$ & \\
\hline 11 & Verify Control Room pressure. & $\begin{array}{l}\text { The differential pressure between } \\
\text { the control room and the outside } \\
\text { atmosphere is maintained at } 0.05 \\
\text { inwg } \pm 0.03 \text { inwg. }\end{array}$ & & \\
\hline 12 & $\begin{array}{l}\text { Verify proper operation of the Non-RCA } \\
\text { HVAC System controls. }\end{array}$ & $\begin{array}{l}\text { The Non-RCA HVAC System operation is } \\
\text { controlled in accordance with Logic } \\
\text { Diagram H-2-89888. }\end{array}$ & $S-1231-100$ & \\
\hline 13 & $\begin{array}{l}\text { Verify proper response of the Non-RCA } \\
\text { HVAC System to receipt of fire } \\
\text { protection signals. }\end{array}$ & $\begin{array}{l}\text { Verify that the Operations Area, } \\
\text { Electrical Equipment Room and } \\
\text { Control Room AHUs shut down upon } \\
\text { receiving a fire signal from the } \\
\text { Fire Alarm Control Panel. }\end{array}$ & $\begin{array}{l}5-1231-402 \\
7.1 .12 .36 \\
7.1 .13 .36 \\
7.2 .9 .36\end{array}$ & \\
\hline
\end{tabular}


HNF-SD-C018H-TS-002, REV. 1

\begin{tabular}{|c|c|c|c|c|}
\hline \multicolumn{5}{|c|}{ Appendix A, Table A-13, Safety Showers (65H) } \\
\hline$\#$ & Test Requirements & Acceptance Criteria & $\begin{array}{l}\text { Completion Regd } \\
\text { By (Document) }\end{array}$ & $\begin{array}{l}\text { Verification } \\
\text { Initials/Date } \\
\end{array}$ \\
\hline \multirow[t]{2}{*}{1} & \multirow[t]{2}{*}{$\begin{array}{l}\text { Safety showers have sufficient } \\
\text { pressure and flow rate. }\end{array}$} & $\begin{array}{l}\text { Safety showers flowrate meets the } \\
\text { minimum requirements of ANSI } \\
\text { standard } 2358.1-1990,30 \mathrm{gpm} \text {. }\end{array}$ & $S-1231-377$ & \\
\hline & & $\begin{array}{l}\text { Eyewash stations are correct as } \\
\text { intended by the vendors instructions } \\
\text { and designs. }\end{array}$ & $S-1231-377$ & \\
\hline
\end{tabular}


HNF-SD-CO18H-TS-002, REV. 1

\begin{tabular}{|c|c|c|c|c|}
\hline \multicolumn{5}{|c|}{ Appendix A, Table A-14, Continuous Air Monitors (70A) } \\
\hline$\#$ & Test Requirements & Acceptance Criteria & $\begin{array}{l}\text { Completion Reqd } \\
\text { By (Document) }\end{array}$ & $\begin{array}{l}\text { Verification } \\
\text { Initials/Date }\end{array}$ \\
\hline 1 & $\begin{array}{l}\text { Verify current calibration of } \\
\text { Continuous Air Monitors }\end{array}$ & $\begin{array}{l}\text { C Must have successfully passed a } \\
\text { WHC OR PNL calibration procedure. }\end{array}$ & & \\
\hline
\end{tabular}

a Action to meet this requirement is to be performed per site approved calibration procedures. 
HNF-SD-CO18H-TS-002, REV. 1

Appendix A, Table A-15, Portable Radiation Instrumentation - 70B

\begin{tabular}{|c|c|c|c|c|}
\hline$\#$ & Test Requirements & Acceptance Criteria & $\begin{array}{l}\text { Completion Reqd } \\
\text { By (Document) }\end{array}$ & $\begin{array}{l}\text { Verification } \\
\text { Initials/Date }\end{array}$ \\
\hline 1 & $\begin{array}{l}\text { Verify current calibration of } \\
\text { Portable Radiation Instrumentation. }\end{array}$ & $\begin{array}{l}\text { Must have successfully passed a } \\
\text { WHC OR PNL calibration procedure. }\end{array}$ & & \\
\hline
\end{tabular}

a Action to meet this requirement is to be performed per site approved calibration procedures. 
HNF-SD-C018H-TS-002, REV. 1

\begin{tabular}{|c|c|c|c|c|}
\hline \multicolumn{5}{|c|}{ Appendix A, Table A-16, PORTAL MONITORS - 70C } \\
\hline$\#$ & Test Requirements & Acceptance Criteria & $\begin{array}{l}\text { Completion Reqd } \\
\text { By (Document }\end{array}$ & $\begin{array}{l}\text { Verification } \\
\text { Initials/Date }\end{array}$ \\
\hline 1 & $\begin{array}{l}\text { Verify current calibration of } \\
\text { Portal Monitors. }\end{array}$ & $\begin{array}{l}\text { a Must have successfully passed a } \\
\text { WHC OR PNL calibration procedure. }\end{array}$ & & \\
\hline
\end{tabular}

a Action to meet this requirement is to be performed per site approved calibration procedures. 
HNF-SD-C018H-TS-002, REV. 1

\begin{tabular}{|c|c|c|c|c|}
\hline \multicolumn{5}{|c|}{ Appendix A, Table A-17, Cooling Water System (95C) } \\
\hline$\#$ & Test Requirements & Acceptance Criteria & $\begin{array}{l}\text { Completion Reqd By } \\
\text { (Document) }\end{array}$ & $\begin{array}{l}\text { Verification } \\
\text { Initials/Date }\end{array}$ \\
\hline 1 & $\begin{array}{l}\text { Verify the following rotating } \\
\text { equipment operates with no evidence } \\
\text { of abnormal operating characteristics } \\
\text { (high vibration, cavitation, high } \\
\text { bearing temperature). }\end{array}$ & $\begin{array}{l}95 C-P-1 \\
95 C-P-2 \\
95 C-F-1 A \\
95 C-F-1 B\end{array}$ & $\begin{array}{l}S-1231-305 S-1231- \\
406 \text { STEP } 7.3 .2 \\
7.3 .13,7.4 .5,7.6 .1 \\
5\end{array}$ & \\
\hline 2 & $\begin{array}{l}\text { Verify proper operation of the } \\
\text { cooling tower heaters. }\end{array}$ & $\begin{array}{l}\text { The cooling tower heaters (95C-E- } \\
2 \mathrm{~A} / 2 \mathrm{~B}) \text { are automatically controlled } \\
\text { from the MCS and are capable of } \\
\text { maintaining a temperature of greater } \\
\text { than freezing point, during } \\
\text { continuous and prolonged operation } \\
\text { of plant and in the shutdown mode. }\end{array}$ & $\begin{array}{l}\text { S.1231-309 S-1231- } \\
406 \text { SECTION } 7.2\end{array}$ & \\
\hline 3 & $\begin{array}{l}\text { Verify proper operation of Flow } \\
\text { proportional sampler SP-95C015. }\end{array}$ & $\begin{array}{l}\text { Capable of pulling and storing a } \\
\text { composite flow proportional sample } \\
\text { of the cooling water blowdown } \\
\text { stream. }\end{array}$ & & \\
\hline 4 & $\begin{array}{l}\text { Verify proper operation of the } \\
\text { Cooling Water System hand switches. }\end{array}$ & $\begin{array}{l}\text { The AUTO/MANUAL position of the } \\
\text { following HS sha11 be initiated at } \\
\text { MCS: } \\
\text { HS- } 95 \mathrm{C} 113 \text {, HS- } 95 \mathrm{C} 114 \text {, HS-95C111, HS- } \\
95 \mathrm{C} 112 \text {, HS-95C031, HS-95C084. }\end{array}$ & $\begin{array}{l}\text { S-1231-406 SECTION } \\
7.8\end{array}$ & \\
\hline 5 & $\begin{array}{l}\text { Verify proper operation of the } \\
\text { Cooling Water System low pressure } \\
\text { alarm. }\end{array}$ & $\begin{array}{l}\text { Alarm PAL-95CO12 is activated at set } \\
\text { point of } 80 \pm 5 \text { psig. }\end{array}$ & & \\
\hline 6 & $\begin{array}{l}\text { Verify proper operation of cooling } \\
\text { water pump low level interlock. }\end{array}$ & $\begin{array}{l}\text { The low level interlock shut down } \\
\text { the cooling water pump when the low } \\
\text { level switch is activated at set } \\
\text { point of } 12.5 \pm 0.5 \text { inches. }\end{array}$ & $\begin{array}{l}\text { S-1231-406 STEP } \\
7.4 .28\end{array}$ & \\
\hline
\end{tabular}


HNF-SD-C018H-TS-002, REV. 1

\begin{tabular}{|c|c|c|c|c|}
\hline \multicolumn{5}{|c|}{ Appendix A, Table A-17, Cooling Water System (95C) } \\
\hline \# & Test Requirements & Acceptance Criteria & $\begin{array}{l}\text { Completion Reqd By } \\
\text { (Document) }\end{array}$ & $\begin{array}{l}\text { Verification } \\
\text { Initials/Date }\end{array}$ \\
\hline 7 & $\begin{array}{l}\text { Verify adequate cooling capacity of } \\
\text { the cooling tower. }\end{array}$ & $\begin{array}{l}\text { The cooling tower can adequately } \\
\text { remove the heat load of the ETF } \\
\text { systems when plant is at full } \\
\text { operating conditions. }\end{array}$ & $\begin{array}{l}\text { S-1231-501 Sect. } \\
7.3 .5 \\
\text { S-1231-502 sect. } \\
7.5-7.8\end{array}$ & \\
\hline 8 & $\begin{array}{l}\text { Verify the cooling water pump motors } \\
\text { operate with normal current draw. }\end{array}$ & $\begin{array}{l}\text { Pumps do not exceed the motor full } \\
10 \text { ad amperage rating of } 115 \text { Amps and } \\
10 \text { Amps for } 95 \mathrm{C}-\mathrm{P}-1 \text { and } 95 \mathrm{C}-\mathrm{P}-2 \\
\text { respectively. }\end{array}$ & $\begin{array}{l}S-1231-305 \text { S-1231- } \\
4067.4 .8,7.6 .30\end{array}$ & \\
\hline 9 & $\begin{array}{l}\text { Verify the cooling tower fan motors } \\
\text { operate with normal current draw. }\end{array}$ & $\begin{array}{l}\text { Fans do not exceed the motor full } \\
\text { load amperage rating of } 45 \text { Amps for } \\
95 C-F-1 A / 1 B \text {. }\end{array}$ & $\begin{array}{l}S-1231-305 \text { S-1231- } \\
4067.3 .6,7.3 .17\end{array}$ & \\
\hline
\end{tabular}


HNF-SD-C018H-TS-002, REV. 1

\begin{tabular}{|c|c|c|c|c|}
\hline \multicolumn{5}{|c|}{ Appendix A, Table A-18, Raw Water System (95H) } \\
\hline$\#$ & Test Requirements & Acceptance Criteria & $\begin{array}{l}\text { Completion Reqd By } \\
\text { (Document) }\end{array}$ & $\begin{array}{l}\text { Verification } \\
\text { Initials/Date }\end{array}$ \\
\hline 1 & $\begin{array}{l}\text { Verify proper operation of system } \\
\text { heat tracing. }\end{array}$ & $\begin{array}{l}\text { All heat trace to system } 95 \mathrm{H} \text { must } \\
\text { be capable of maintaining a } \\
\text { temperature above freezing. }\end{array}$ & $\begin{array}{l}\text { S-1231-338 } \\
\text { Sect. } 6.0\end{array}$ & \\
\hline
\end{tabular}

